\title{
DINÁMICA PRODUCTIVA Y DESARROLLO EN LAS ÁREAS RURALES
}

\author{
Antonio Vázquez-Barquero \\ Universidad Autónoma de Madrid \\ España
}


Panorama Económico, Vol. 25 - No. 3 (Julio - Septiembre de 2017), pp. 289-296

Antonio Vázquez-Barquero

\title{
Dinámica productiva y desarrollo en las áreas rurales
}

\section{Resumen}

El desarrollo de las áreas rurales se encuentra sometido a importantes desafíos. En éste sentido, la visión de que el desarrollo agrícola y el desarrollo territorial son complementarios se ha fortalecido recientemente, si se busca la diversificación productiva y la sostenibilidad económica y social. Por otra parte, la identidad cultural de los territorios está en la base del sistema productivo y condiciona su evolución en el tiempo, permitiendo la diferenciación y la consolidación de ventajas comparativas adquiridas con la introducción de innovaciones. Por último, los procesos de cambio requieren el fortalecimiento de las instituciones: impulsando la descentralización y la coordinación entre los distintos niveles de la administración pública; y erradicando la corrupción que no solo reduce la eficiencia y aumenta la desigualdad, sino que debilita el proceso de acumulación y, por lo tanto, el progreso económico y social.

Palabras clave: desarrollo económico, áreas rurales, desarrollo territorial, desarrollo sostenible.

Clasificación JEL: O12, O13, 018, Q01

\section{Productive dynamics and development in rural areas}

\begin{abstract}
The development of rural areas face enormous challenges. On this regard, the role of agricultural and territorial development as complementaries increased substantially if considering productive diversification and sustainable development. On th other hand, culture in territories is key for productive systems and determines its evolution towards comparative advantages. Last, processes of change need solid institutional settings: towards descentralization and coordination among different levels of the public administration and eliminating corruption that reduce efficiency and augment inequality.

Keywords: Economic development, rural areas, territorial development, sustainable development.

JEL Classification: O12, O13, 018, Q01
\end{abstract}

\section{Dynamique de production et développement en milieu rural}

\section{Résumé}

Le développement des aires rurales ont plusieurs défis. Les développements agraires et territoriaux vont récemment vers la diversification agricole et développement soutenable. L'identité culturelle des territoires se base sur le système de production, donc son Evolution selon le temps. Des innovations sont introduites et acquises. Le changement requière le renforcement des institutions par la décentralisation et agencement avec différents paliers gouvernementaux. Ceci A comme effet secondaire d'éliminer les effets nocifs de la corruption.

Mots-clés: Devellopement économique, zone rurale, devellopment territoriale, devellopment soutenable Nomenclature JEL: O12, O13, 018, Q01 


\section{Dinámica productiva y desarrollo en las áreas rurales}

INFORMACIÓN DEL ARTÍCULO

Recepción de artículo: 30/04/2017

Concepto de evaluación: 25/05/2017

Aceptación de artículo: 29/06/2017
Antonio Vázquez-Barquero* Universidad Autónoma de Madrid España

La globalización y las nuevas tecnologías de la información y de las comunicaciones están transformando el sistema productivo y el entorno institucional de las áreas rurales. Su dinámica económica es el resultado de la aplicación de los recursos naturales, de los factores productivos y de las innovaciones tecnológicas, mediante las inversiones que realizan los propietarios agrarios y las empresas locales. Las decisiones se toman en un entorno institucional y cultural que asegura los derechos de propiedad, lo que da confianza para afrontar los riesgos que plantea la globalización.

El aumento de la diversidad y la diferenciación de los sistemas productivos se aprecia de forma particular en las áreas rurales que se enfrentan a la crisis de la agricultura tradicional, la despoblación, la falta de infraestructuras básicas, el deterioro del medio ambiente y el incremento de la competencia. El medio rural afronta el reto en un mundo en el que se está produciendo una nueva división internacional del trabajo. En el nuevo marco, las áreas rurales están diversificando sus actividades productivas, superando el paradigma que identificaba las actividades agrícolas con las áreas rurales, y las actividades industriales y de servicios con las áreas urbanas.

De hecho, se está produciendo un proceso de diversificación espontánea cuando aumenta la renta dado que amplia y diferencia la demanda, y cuando se reducen los costes de transporte fruto de la mejora del sistema de comunicaciones. Así, sobre todo, desde principios de siglo, la atracción de recursos humanos y financieros está promoviendo el desarrollo de las actividades de servicios (claramente, en lo que atañe al turismo $\mathrm{y}$ al comercio), el fortalecimiento de las actividades industriales en las localidades y

\footnotetext{
* Autor para correspondencia

Correos electrónicos: vasquez-barquero@uam.es
} 
pequeñas ciudades, la diferenciación de los productos agrarios, y la ampliación de los mercados. En el proceso de transformación productiva y de integración económica de las regiones rurales juegan un papel destacado las elites económicas, sociales e institucionales, cuando se toman las decisiones de inversión.

En los territorios rurales se advierte una gran diversidad de situaciones. Por un lado, las regiones remotas, como las regiones amazónicas, formadas por territorios aislados y con sistemas productivos frágiles, frecuentemente, tienen baja densidad de población y cuentan con unos recursos naturales y un patrimonio histórico y cultural que se deteriora paulatinamente. En las regiones que están físicamente apartadas pero que cuentan con un potencial de desarrollo, como por ejemplo en la región del Orinoco en Venezuela, los actores locales pueden hacer uso de los recursos y de las capacidades existentes e integrar los territorios en la economía internacional. En las áreas rurales con capacidad innovadora, como en Andalucía, que se integran en el sistema económico internacional a través de redes (productivas, comerciales, tecnológicas), la capacidad emprendedora y la flexibilidad de sus instituciones permiten generar proyectos con una dinámica propia (Rodríguez Cohard et al., 2017). Por último, las áreas con producciones muy apreciadas en los mercados tienen una identidad territorial reconocida a nivel internacional, como les ocurre a los territorios productores de vino en Chile y Argentina y al eje cafetero en Colombia (Sanz-Cañada y Muchnick, 2016).

Las fuerzas locales del desarrollo facilitan la dinámica productiva y la diferenciación de la producción (Vazquez, 2005). Así, la organización, a través de las redes de empresas y el cooperativismo, facilita la actividad productiva. Por un lado, los clústeres propician el intercambio de información entre los propietarios de las explotaciones agrarias y entre las empresas manufactureras, como muestra la Empresa Colineal del mueble en Ecuador, lo que estimula la difusión del conocimiento tecnológico y reduce los costes de los intercambios dentro de la red. Además, las cooperativas, tan difundidas en América Latina, con más de mil trescientas en Chile, refuerzan los emprendimientos a través de la participación ciudadana.

Enlasúltimasdécadas, sehaincrementado la adopción de innovaciones en las actividades rurales, introduciendo nuevas técnicas de producción con maquinaria que incorporan conocimiento técnico, lo que facilita la diferenciación de los productos agrarios tradicionales y el surgimiento de nuevos productos. Es más, en algunos territorios han aumentado las capacidades tecnológicas y los vínculos de las empresas, como sucede en el caso de las empresas argentinas de maquinaria agrícola (Mochi, 2012). Así pues, la difusión de las innovaciones por el tejido productivo permite la diferenciación de la producción en las explotaciones agrarias y en las redes de empresas rurales.

Los procesos de desarrollo rural se generan de acuerdo con las instituciones y las raíces culturales de los territorios (North, 1990; Ostrom. 2005), ya que las iniciativas y decisiones de inversión se apoyan en las normas formales e informales propias de las localidades y regiones. La dinámica productiva de las áreas rurales está fuertemente condicionada por las leyes y la constitución, pero también por el sistema social, el capital social civil y público 
y por el tejido cultural. Así pues, los procesos de desarrollo rural se apoyan en un sistema institucional complejo, entre cuyos factores figuran las estructuras familiares que condicionan la organización de la producción, la identidad local de la población que los vincula al territorio, y la importancia que se concede a la capacidad emprendedora. Cuando ello genera confianza entre los propietarios y los emprendedores, se facilita el funcionamiento de las empresas y su presencia en los mercados.

Así pues, en un escenario, como el actual, caracterizado por la globalización y la ampliación de los mercados, la diversificación de las actividades productiva de las áreas rurales, el aumento de las actividades de servicios y las nuevas formas de integración exterior contribuyen, de manera singular, al desarrollo diferenciado de las localidades $\mathrm{y}$ de los territorios rurales (Saraceno, 2006). Además, los procesos de desarrollo se fortalecen en aquellos territorios que disponen de infraestructuras de transporte y de comunicaciones, ya que la mejora de la accesibilidad amplía sus mercados y estimula la competencia de las explotaciones y de las empresas locales.

Tradicionalmente, dada la dispersión de los recursos locales, las áreas rurales se han enfrentado con grandes desventajas para atraer inversiones $y$ visitantes debido a los costes que implican las deficiencias del sistema de transporte. Las infraestructuras de transporte y comunicaciones son clave para el desarrollo de la actividad productiva rural, asociada con el abastecimiento de materias primas y de inputs intermedios que impulsen la transformación de las actividades locales, entre las que son, cada vez, más importantes las que incorporan nuevas tecnologías y la expansión de los servicios.

En los territorios más dinámicos del medio rural, la agricultura ha dejado de ser la principal fuente de riqueza, mientras que las actividades vinculadas con los productos agroalimentarios, el textil, el calzado y los muebles han aumentado su peso en los municipios rurales, lo mismo que las nuevas actividades de servicios turísticos y de ocio. En particular, desde los inicios del siglo se están fortaleciendo las industrias culturales, lo que favorece la recuperación de los territorios, cuya economía tradicional se ha debilitado. Según la Unesco, la cultura de los territorios la forman el conjunto de las artes, de los modos de vida, las tradiciones y creencias. Así el patrimonio natural y cultural se está convirtiendo en un estímulo para el desarrollo del turismo a través de las rutas temáticas y de los itinerarios culturales.

Pero, la dinámica productiva de las regiones rurales en las últimas décadas, varía de unos territorios a otros en función de la evolución de la demanda de sus productos en los mercados, ya que el aumento de la competencia favorece la diferenciación de la producción y la integración de los sistemas productivos. En los territorios tropicales, es frecuente encontrarse ante situaciones de utilización ineficiente de los recursos naturales e incluso de su destrucción, como es el caso de la deforestación sistemática en muchos lugares de la cuenca del Amazonas. Pero, cuando existen buenas prácticas como en el caso de la Comunidad Indígena de San Juan Nuevo Parangaricutiro (SJN), de la región Purépecha del Estado de Michoacán, la empresa forestal ha gestionado, durante veinticinco años, los recursos existentes, 
siendo un estímulo para el desarrollo autosostenido del territorio (Vázquez, 2016).

La nueva dinámica del sistema productivo que se produce como consecuencia de la integración creciente de las economías y de los mercados está creando una nueva geografía del desarrollo en todo tipo de territorios, y en particular en los territorios innovadores. Elaumento dela competencia en los mercados ha dado lugar a respuestas diferentes en las áreas rurales, ya que las condiciones económicas, tecnológicas y culturales no son uniformes en todos los territorios, lo que explica las diferencias en la productividad de las explotaciones agrarias y de las empresas, en la adopción y difusión de las innovaciones en los sistemas productivos, y en los sistemas institucionales. Todo ello abre un espacio para las políticas públicas.

En estos entornos tan diversos, el desarrollo rural se propone objetivos múltiples que hagan sostenibles los procesos de crecimiento y cambio estructural, como son los objetivos económicos, sociales y medioambientales. Pero, dada la incapacidad de alcanzar todos los objetivos al mismo tiempo, los territorios suelen establecer prioridades que se van adaptando en función del escenario de desarrollo en el que se toman las decisiones. Así, en las regiones que son reserva de los recursos naturales, como Tayrona en Colombia, se prioriza el medioambiente, al mismo tiempo que se trata de alcanzar objetivos económicos y sociales facilitando ciertas actividades turísticas específicas. A su vez, en las regiones agroindustriales, como en los territorios productores de vino en Chile y en Argentina, se priorizan los objetivos económicos manteniendo la dimensión social y medioambiental.

En resumen, el desarrollo de las áreas rurales está sometido a importantes desafíos. Ante todo, cobra cada vez más fuerza la visión que entiende que en las áreas rurales el desarrollo agrícola y el desarrollo territorial son complementarios, si se quiere favorecer la diversificación de la actividad productiva y la sostenibilidad económica y social. Por otro lado, la identidad cultural de los territorios está en la base del sistema productivo y condiciona su evolución en el tiempo, lo que permite la diferenciación de la producción y el reforzamiento de las ventajas comparativas adquiridas con la introducción de innovaciones. Por último, los procesos de cambio requieren el fortalecimiento de las instituciones: impulsando la descentralización y la coordinación entre los distintos niveles de las administraciones; estimulando las relaciones entre las empresas y los actores públicos; y erradicando la corrupción que no solo reduce la eficiencia y aumenta la desigualdad, sino que debilita el proceso de acumulación y, por lo tanto, el progreso económico y social.

\section{REFERENCIAS BIBLIOGRÁFICAS}

Mochi, S. (2012). Aglomerados productivos, institucionalidad y competitividad en las Pymes. Análisis de casos de Maquinaria Agrícola y Forestal. Industrializar Argentina, 17: 26-32.

North, D. C. (1990). Institutions, Institutional Change and Economic Performance. Cambridge University Press, Cambridge. 
Ostrom, E. 2005. Understanding Institutional Diversity. Princeton University Press, New Jersey, EE UU.

Rodríguez Cohard, J.C., Sánchez Martinez, J.D. y Gallego Simón, V.J. (2017): The upgrading strategy of olive oil producers in Southern Spain: origin, development and constraints. Rural Society, 26 (1): 30-47.

Sanz-Cañada, J. y Muchnick, J. (2016): Geographies of Origin and Proximity: Approaches to Local Agro-Food Systems. Culture \& History Digital Journal, 5 (1): 2-19.

Saraceno, E. (2006). "Políticas rurales de la Unión Europea y proyectos territoriales de identidad cultural". Trabajo presentado en el Seminario "Territorios con Identidad Cultural", abril. Cuzco, Perú.

Vázquez Barquero, A. (2005). Las nuevas fuerzas del desarrollo. Antonio Bosch editor, Barcelona.

(2016). Cambio de Modelo de Desarrollo de los Territorios Innovadores: la complejidad del proceso. Revista Mexicana sobre Desarrollo Local, 1: 15-26.

\section{Para citaciones:}

Vasquez-Barquero, A. (2017). Dinámica productiva y desarrollo en las áreas rurales. Panorama Económico, 25, 3, pp. 289-296.

\section{AUTOR}

Antonio Vázquez-Barquero

Catedrático y Profesor Emérito de la Universidad Autonóma de Madrid (España). Visiting Fellow en Yale University (USA) y enseñado en la Universidad Católica de Chile, la Universidad de los Andes (Colombia), Universidad Central de Venezuela, Universidad de San Martin (Argentina), Universidad de Cádiz (España), Universidad de Insubria (Italia), entre otras. Entre sus líneas de investigación se destacan: desarrollo endógeno, organización espacial de la producción, innovación y cambio tecnológico, y política de desarrollo local. 


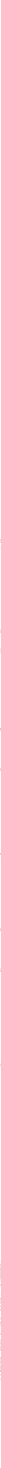

